\title{
Spectroscopic Information from Angular Correlations and Transfer Reactions.
}

\section{A. Strazzeri}

Istituto di Fisica Nucleare dell'Università - Catania

Istituto Nazionale di Fisica Nucleare - Sezione di Catania

Centro Siciliano di Fisica Nucleare e di Struttura della Materia - Catania

(Nuovo Cimento, 39 A, 193 (1977))

The following corrections should be made:
p. 198, last line
errata
corrige

$\sum_{I_{\mathrm{b}}} \quad \sum_{I_{\mathrm{b}} J}$

p. 201, first line before eq. (14)

eq. (9)

eq. (10)

p. 201, eq. (14)

$(-)^{L_{\mathrm{e}}^{\prime}}(2 l+1) \quad(-)^{L_{\mathrm{c}}+l}(2 l+1)^{\frac{1}{l}}$

p. 201, second line below eq. (14)

$W(\ldots)$

$(-)^{L_{\mathrm{c}}+L_{\mathrm{c}}^{\prime}+l} W(\ldots)$

p. 201, second line of eq. (I5)

$S_{N L_{\mathrm{c}}}^{\frac{1}{2}}$

$(-)^{L_{\mathrm{c}}^{\prime}}(2 l+1)^{\frac{1}{2}} S_{N L_{\mathrm{c}}}^{\frac{1}{2}}$

p. 209 , eq. $(23 a)$

$(2 l+1)$

$(2 l+1) / 3$ 